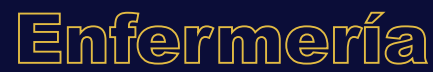

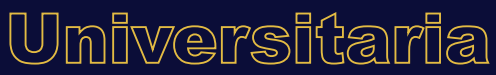

\section{Diferencias del automanejo en personas con enfermedades crónicas en población peruana}

\section{Differences in self-management among persons with chronic diseases in Peruvian populations}

\section{Diferenças na autogestão em pessoas com doenças crônicas na população peruana}

\section{D.J. Malibrán-Luque ${ }^{a 1}{ }^{*}$, M.S. Piñones-Martínez ${ }^{\text {b2 }}$, T. Gutiérrez-Gómez ${ }^{\mathrm{c} 2}$, R.P. Rivero-Álvarez ${ }^{\mathrm{d} 3}$, M.G. Lévano-Cárdenas ${ }^{\mathrm{e}}$, G.J. Vejarano-Campos ${ }^{\mathrm{f}_{4}}$}

ORCID

${ }^{\mathrm{a}} 0000-00033380-1528$

d0000-0002-9804-7047

b $0000-0002-1013-7700$

e $0000-0002-1451-0365$

c 0000-0001-5522-4237

f $\underline{0000-0001-5084-3872}$

${ }^{1}$ Universidad Autónoma de Tamaulipas, Facultad de Medicina Dr. Alberto Romo Caballero, Tampico, Tamaulipas, México

${ }^{2}$ Universidad Autónoma de Tamaulipas, Facultad de Enfermería, Tampico, Tamaulipas, México

3 Universidad César Vallejo, Escuela de Enfermería, Lima, Perú

${ }^{4}$ Instituto Nacional de Enfermedades Neoplásicas, Área de Investigación del Departamento de Enfermería, Lima, Perú

Recibido: 31 julio 2020

Aceptado: 25 abril 2021

RESUMEN

Introducción: El automanejo en personas con alguna condición crónica no es una opción, realizarlo adecuadamente marca la diferencia en la calidad de vida de las personas con una enfermedad crónica, como son cáncer, diabetes e hipertensión arterial. Existe aún muy poco

*Autor para correspondencia. Correo electrónico: dmalibran@docentes.uat.edu.mx https://doi.org/10.22201/eneo.23958421e.2021.1.951

1665-7063/@ 2021 Universidad Nacional Autónoma de México, Escuela Nacional de Enfermería y Obstetricia. Este es un artículo Open Access bajo la licencia CC BY-NC-ND (http://creativecommons.org/licenses/by-nc-nd/4.o/). 
conocimiento sobre similitudes o diferencias del automanejo entre estos tres grupos de individuos.

Objetivo: Describir el automanejo a través del análisis de las diferentes dimensiones en tres grupos de personas con diabetes, hipertensión y cáncer.

Método: Estudio transversal-comparativo con $n=562$ personas: 160 con cáncer, 187 con diabetes y 215 con hipertensión. Muestreo no probabilístico intencional. Se aplicó el instrumento Partners in Health (PIH). Se utilizó la estadística descriptiva y no paramétrica a través de la prueba de Kruskall Wallis para comparar tres muestras independientes, se calculó el efecto de la diferencia y la potencia estadística.

Resultados: El índice general de automanejo y sus dimensiones en los tres grupos están por debajo de la media general respectivamente. No se encontraron diferencias entre los grupos según su diagnóstico médico (diabetes, hipertensión y cáncer). $\rho\langle 0.001$; (1- $\beta\rangle .80$ y d > .20).

Discusión y Conclusiones: Las personas con alguna condición crónica presentan un automanejo inadecuado, no se identifican diferencias en el automanejo global y sus dimensiones según diagnóstico médico. En consecuencia, se hace relevante la implementación de programas genéricos que promueven el automanejo, sobre todo en el primer nivel de atención, además se debe incrementar las investigaciones en Latinoamérica, con mayor atención en personas con cáncer.

Palabras clave: Autocuidado; automanejo; cáncer; diabetes mellitus; hipertensión; Perú.

\section{ABSTRACT}

Introduction: An appropriate self-management by persons living with chronic conditions such as cancer, diabetes, or hypertension, can make a big difference in terms of quality of life. Therefore, it is important to enhance our knowledge regarding how persons suffering from any of these chronic diseases self-manage their condition.

Objective: To describe how Peruvian persons living with cancer, diabetes, or hypertension, self-manage their condition.

Méthod: This is a transversal-comparative study on a sample of $n=562$ Peruvian persons: 160 with cancer, 187 with diabetes, and 215 with hypertension. The sampling was probabilistic and intentional. The Partners in Health (PIH) instrument was used. Descriptive and non-parametric statistics were calculated. The Kruskall Wallis test was used to compare three independent sample types. The difference effects and statistical power were also calculated.

Results: The general self-management index and its related dimensions in the three groups were below the general mean respectively. No differences were found between groups in terms of clinical diagnosis (cancer, diabetes mellitus, hypertension). $\rho<0.001 ;(1-\beta>.80$ and d>0.20).

Discussion and Conclusions: Persons with chronic conditions demonstrate inadequate self-management. No differences were found based on the medical diagnosis. Consequently, it is imperative to implement programs aimed at improving self-management, particularly within the first level of attention. More research on the issue needs to be conducted in Latin America, especially regarding persons suffering from cancer.

Keywords: Self-care; self-management; cancer; diabetes mellitus; hypertension; Peru. 


\section{RESUMO}

Introdução: A autogestão em pessoas com alguma condições crônicas não é uma opção, realizá-la adequadamente marca a diferença na qualidade de vida de pessoas com uma doença crônica, como câncer, diabetes e hipertensão arterial. Ainda há pouco conhecimento sobre semelhanças ou diferenças na autogestão entre esses três grupos de indivíduos.

Objetivo: Descrever a autogestão por meio da análise das diferentes dimensões em três grupos de pessoas com diabetes, hipertensão e câncer.

Método: Estudo transversal comparativo com $n=562$ pessoas: 160 com câncer, $187 \mathrm{com}$ diabetes e 215 com hipertensão. Amostragem não probabilística intencional. Foi aplicado o instrumento Partners in Health (PIH). Foram utilizadas estatísticas descritivas e não paramétricas por meio do teste de Kruskall Wallis para comparar três amostras independentes, calculou-se o efeito da diferença e a potência estatística.

Resultados: O índice geral de autogestão e suas dimensões nos três grupos estão abaixo da média geral, respectivamente. Não foram encontradas diferenças entre os grupos de acordo com o diagnóstico médico (diabetes, hipertensão e câncer). $\rho<0.001$; (1- $\beta>$ >.80 y d >.20).

Discussão e Conclusões: Pessoas com condição crônica apresentam autogestão inadequada, não se identificam diferenças na autogestão global e suas dimensões conforme o diagnóstico médico. Consequentemente, torna-se relevante a implantação de programas genéricos que promovam a autogestão, principalmente no primeiro nivel de atenção, aliás devem-se aumentar as pesquisas na América Latina, especialmente em pessoas com câncer.

Palavras chave: Autocuidado; autogestão; câncer; diabetes mellitus; hipertensão; Peru.

\section{INTRODUCCIÓN}

De acuerdo con la Organización Mundial de la Salud (OMS), las condiciones crónicas de salud agrupan enfermedades de larga duración y de progresión lenta. Además, representan uno de los mayores retos que enfrenta el sistema de salud por su magnitud, su contribución a la mortalidad general, la incapacidad prematura, la complejidad, así como el costo elevado de su tratamiento. Entre las enfermedades de mayor prevalencia se encuentran la diabetes, hipertensión y cáncer, entre otras ${ }^{1}$, siendo estas tres las de mayor prevalencia en América Latina, como es el caso de Perú.

Desde el año 2000 el cáncer se ha constituido en la segunda causa de muerte en Perú (17\%), existe además un incremento significativo en el número de casos nuevos²; aunado a ello, la prevalencia de diabetes entre adultos de 25 años o más fue del 7.0\%; de estas personas 40\% no había sido diagnosticada3. En la Encuesta Demográfica y de Salud Familiar 2017 (ENDES) se encontró que 13.6\% de la población de 15 y más años de edad tenía presión arterial alta; en el 2016 fue 12.7\%. De acuerdo con el sexo, los hombres son más afectados (16.6\%) que las mujeres (10.7\%), relación similar con respecto a 2016 (hombres $15.6 \%$ y mujeres $9.9 \%$ ) 4.5 .

Con los avances en las ciencias médicas se han modificado los resultados de muchas enfermedades crónicas, lo que se traduce en menos años de vida saludable perdidos, prestando mayor interés a estrategias o métodos que permitan mantener la independencia y calidad de vida de todo individuo con una o más enfermedades crónicas. Esta nueva forma de abordar la cronicidad pone en el centro de la atención médica al mismo paciente, dándole un rol principal en su propio cuidado en coordinación con los prestadores de atención de salud; es decir, tenemos un nuevo paradigma de atención centrado en el paciente ${ }^{6}$, donde uno de los objetivos principales es promover el automanejo de su condición crónica. 
El automanejo se define comúnmente como aquellas estrategias que las personas realizan para vivir bien, incluido el cuidado profesional médico, la responsabilidad de sus roles sociales y emocionales ${ }^{7}$. Esta definición se basa en el modelo de Corbin y Strauss ${ }^{8}$ en el que destacan las demandas generalizadas que se imponen a las personas que viven con una afección a largo plazo9. Ubicándonos en el contexto de la cronicidad, teniendo en cuenta las condiciones de la vida misma, que fluctúan y cambian con el tiempo, el automanejo es un proceso continuo y de por vida con diferentes demandas, varía en el momento del diagnóstico, una vez que se ha alcanzado una nueva normalidad y cuando el envejecimiento o la multimorbilidad complican la vida ${ }^{10,11}$.

Actualmente existe una diversidad de marcos conceptuales y teorías de automanejo, a menudo, la mayoría describen los mecanismos subyacentes del apoyo como las intervenciones involucradas. Por ejemplo, Vernooij et al. ${ }^{12}$ identificaron componentes críticos que mejoran la eficacia de las intervenciones de automanejo, Pearce et al. ${ }^{13}$ desarrollaron una taxonomía de apoyo a la autogestión para especificar componentes y modos de administración; Asa Audulv et al. ${ }^{14}$ proponen una taxonomía en el automanejo de personas con afecciones neurológicas; Schulman-Green et al..$^{15}$ describen una taxonomía de conceptos, actividades como tareas a considerar en el automanejo. Por su parte, Ryan ${ }^{16}$ desarrolla una teoría de rango medio describiendo al automanejo dentro de un contexto, proceso y resultado. Esta diversidad de conceptos muchas veces dificulta la posibilidad de unificar recursos tales como: términos estandarizados, herramientas de medición para evaluar programas que promueven el automanejo y guías para el desarrollo de la intervención, entre otros.

Esta investigación se centra en los marcos de automanejo diseñados por investigadores de la Universidad Flinders ${ }^{17,18}$, quienes además de describir el concepto de automanejo que incorpora los conceptos antes mencionados y de otros teóricos como Gruman, Lorig ${ }^{19}$, Io plasman en un programa genérico para promoverlo. De igual forma, evalúan dicho comportamiento en cualquier persona con alguna condición crónica, identificando los siguientes principios o características del automanejo, como se observa en la tabla 1.

\section{Tabla 1. Caracteristicas del automanejo}

Conocimiento de su estado de salud y su enfermedad

Adopción de un plan de cuidados acordado en asociación con profesionales de la salud, cuidadores y otros ayudantes

Participar activamente en la toma de decisiones con profesionales de la salud, cuidadores y otros ayudantes

Supervisión y control de los signos y síntomas de la condición

Controlar el impacto de la condición en el funcionamiento físico, emocional, ocupacional y social

Adoptar estilos de vida que aborden los factores de riesgo y promuevan la salud centrándose en la prevención y la intervención temprana

Tener acceso y confianza de usar servicios de apoyo

Fuente: Battersby MW. 2005; Battersby MW, et al. 2003

El presente estudio tiene como propósito describir el automanejo en personas con enfermedades crónicas (diabetes, hipertensión y cáncer) por ser las de mayor prevalencia en Perú, el cual permita identificar áreas a mejorar en este comportamiento, así como diferencias y similitudes entre los tres grupos, comportamiento de automanejo que se esperaría en cualquier persona, sea cual sea su diagnóstico ${ }^{17,18}$. Esto con el fin de generar evidencia científica que permita fundamentar la necesidad de implementar programas de automanejo en nuestro sistema de salud, focalizar mejor las áreas a ser 
abordadas de manera conjunta, cualquiera sea la enfermedad diagnosticada y cuales requerirían un abordaje más especializado; sobre todo en el primer nivel de atención, donde se deben manejar programas genéricos para abordar a la población con cualquier condición crónica, garantizando mayor accesibilidad a este tipo de educación y por ende mayor probabilidad de una mejor calidad de vida.

\section{MÉTODOS}

Se trata de un diseño transversal, comparativo. Esta investigación forma parte de un proyecto Matriz binacional: Red de automanejo de enfermedades crónicas ${ }^{20}$ realizado por la Facultad de Enfermería Tampico de la Universidad Autónoma de Tamaulipas y socios de la Red de las sedes seleccionadas: Ministerio de Salud-Dirección de Servicios de Salud Lima-Norte y Departamento de Enfermería del Instituto Nacional de Enfermedades Neoplásicas de Perú. Se realizó entre los meses de octubre de 2016 y diciembre de 2017.

La población de estudio fueron personas con diagnóstico de cáncer, usuarios de servicios de consulta externa y quimioterapia del Instituto Nacional de Enfermedades Neoplásicas en Lima, Perú; así como usuarios diagnosticados con diabetes o hipertensión de los centros de salud Lima-Norte del Ministerio de Salud.

Se realizó un muestreo no probabilístico intencional realizado según las exigencias de participación del programa Tomando Control de su Salud de la Universidad de Stanford ${ }^{20}$, el cual debe ser realizado por dos personas previamente certificadas como Líderes para implementar el programa y cada grupo participante no debe ser menor a 12 ni mayor a 16 integrantes. Es así como se conformaron grupos de participantes según corresponde al total de la muestra de personas con alguna condición crónica.

Los encuestados se seleccionaron en la base de datos de las mediciones del programa realizado en 2016-17 en la sede de la Red. La muestra quedó conformada por un total de 562 personas, distribuidas en: 160 personas con cáncer, 187 personas con diabetes y 215 personas con hipertensión.

Los criterios de inclusión fueron: personas con diagnóstico de cáncer, diabetes o hipertensión (si presentaba dos de las tres enfermedades se registró el primer diagnóstico), tener 18 o más años de edad, no presentar problemas cognitivos (orientado en tiempo, persona y lugar), ser usuarios ambulatorios (consulta externa, quimioterapia), de los centros de salud del Ministerio de Salud. Los criterios de exclusión fueron: que no quisieran participar en el estudio, personas con diagnóstico de cáncer, diabetes o hipertensión como enfermedad concomitante simultánea al embarazo, personas con diagnóstico de cáncer en etapa terminal o con cuidados paliativos.

Se utilizó el Instrumento de Automanejo Escala de Compañeros en Salud, por sus siglas en inglés Partners in Health Scale (PHS) ${ }^{18}$. Este instrumento, en su versión original, fue elaborado y validado por un equipo de investigadores de la Universidad Flinders de Australia; el cual tiene como objetivo evaluar el comportamiento del automanejo en personas con alguna condición crónica. La confiabilidad del instrumento reportó un alpha de Cronbach de $0.82^{18}$. Consta de 12 reactivos, con una escala tipo Likert que va desde 0 hasta 8 , donde 0 indica bajo automanejo y 8 indica alto automanejo, fue validado previamente por el equipo de investigación del presente proyecto en población peruana donde reportó un alpha de Cronbach de 0.72. Evalúa 3 dimensiones: conocimiento (preguntas 1 y 2), adherencia al cuidado (ítems 3, 4, 5, 6, 7, 8 y 12) y manejo del impacto psicológico, físico y social de la condición crónica (preguntas 9, 10, 11). La fiabilidad estadística para cada dimensión fue 0.78 para conocimiento, 0.96 para adherencia a cuidados médicos y 0.70 para manejo de salud ${ }^{22}$. 
Además de estas 3 dimensiones el instrumento permite evaluar las 6 características descritas en el comportamiento del automanejo: conocimiento (preguntas 1 y 2), participación (preguntas 3 y 4), servicios de apoyo (preguntas 5 y 6), supervisión y respuesta (preguntas 7 y 8), impacto físico, emocional y social (se evalúa con las preguntas 9, 10,11) y estilo de vida (pregunta 12), descritas en la tabla 2.

Tabla 2. Dimensiones y caracteristicas del instrumento

\begin{tabular}{|c|c|c|}
\hline Dimensión & Preguntas & Características \\
\hline \multirow{2}{*}{$\begin{array}{l}\text { Conocimiento } \\
\text { (0-16 puntos) }\end{array}$} & 1. Conocimiento de la enfermedad & \multirow{2}{*}{$\begin{array}{l}\text { Conocimiento acerca de su } \\
\text { condición crónica y plan de } \\
\text { tratamiento }\end{array}$} \\
\hline & $\begin{array}{l}\text { 2. Conocimiento de tratamiento de las } \\
\text { enfermedades }\end{array}$ & \\
\hline \multirow{7}{*}{$\begin{array}{l}\text { Adherencia al cuidado } \\
\text { médico } \\
\text { (0-56 puntos) }\end{array}$} & 3. Tomar la medición prescrita & \multirow{7}{*}{$\begin{array}{l}\text { Participa en la toma } \\
\text { de decisiones de su } \\
\text { automanejo, tiene acceso } \\
\text { a algún servicio de apoyo } \\
\text { y maneja en particular la } \\
\text { supervisión y respuesta } \\
\text { de signos y síntomas y } \\
\text { adopta estilos de vida que } \\
\text { promueven la salud }\end{array}$} \\
\hline & 4. Participación en las decisiones & \\
\hline & 5. Organizar y asistir a las citas & \\
\hline & $\begin{array}{l}\text { 6. Comprensión de las necesidades de revisar y } \\
\text { registrar síntomas }\end{array}$ & \\
\hline & 7. Comprobación y anotado de los síntomas & \\
\hline & $\begin{array}{l}\text { 8. Conocimiento de lo que hay que hacer } \\
\text { cuando los síntomas empeoran }\end{array}$ & \\
\hline & $\begin{array}{l}\text { 12. Realiza actividades que promueven una vida } \\
\text { saludable }\end{array}$ & \\
\hline \multirow{3}{*}{$\begin{array}{l}\text { Manejo del impacto } \\
\text { psicológico, físico y social } \\
\text { de la condición crónica } \\
\text { (0-24 puntos) }\end{array}$} & $\begin{array}{l}\text { 9. Trata las consecuencias de las condiciones } \\
\text { crónicas en el aspecto emocional }\end{array}$ & \multirow{3}{*}{$\begin{array}{l}\text { Maneja los efectos de } \\
\text { su condición crónica con } \\
\text { relación a la actividad física } \\
\text { emocional y social }\end{array}$} \\
\hline & $\begin{array}{l}\text { 10. Trata las consecuencias de su condición } \\
\text { crónica en el aspecto físico }\end{array}$ & \\
\hline & $\begin{array}{l}\text { 11. Trata las condiciones crónicas de su } \\
\text { condición crónica en la vida social }\end{array}$ & \\
\hline
\end{tabular}

Nota: El instrumento permite evaluar el comportamiento del automanejo a través de la medición de tres dimensiones y seis características distribuidas entre las doce preguntas del cuestionario.

La interpretación de los resultados es a mayor puntaje, mejor automanejo. La sumatoria global va en rangos de 0 a 96; con los siguientes puntajes: conocimiento de 0-16; cuidados médicos de 0-56; manejo del impacto físico, emocional y social de 0-24. El instrumento evalúa el comportamiento desde la perspectiva del proveedor de salud al ser él quien realiza las preguntas y da un puntaje según su criterio; también puede ser aplicado a la misma persona con alguna condición crónica, siendo ella la que da el puntaje a la pregunta. Para este estudio se considera la perspectiva de la persona con diagnóstico de enfermedad crónica, ya que esta evaluaba cada dimensión. Cabe señalar que dicho instrumento es reconocido por su utilidad y validez en varios países ${ }^{23-25}$.

La encuesta fue aplicada por estudiantes del último año de Enfermería, previamente capacitadas. La duración de la aplicación fue de 30 minutos, realizada en los mismos servicios de salud en un ambiente acondicionado sin ruido y con privacidad. No se presentaron problemas en su aplicación.

Para el análisis estadístico se realizó la exploración de datos, mostrando la variable de estudio no normalidad, ni homogeneidad de varianzas (prueba de Kolmogorov Smirnov y prueba de Leven p $\leq$ 0.05). Para el análisis de diferencias entre los tres grupos se utilizó la técnica de Kruskall Wallis, a través del paquete estadístico SPSS versión 26. Con el programa $G^{*}$ Power se calculó el efecto de la 
diferencia (d) con el fin de explicarla en el automanejo entre grupos según el diagnóstico: cáncer o diabetes o hipertensión; también, si de acuerdo al diagnóstico esta diferencia es relevante con puntajes que van de pequeño $=0.10$, mediano $=0.30$ y grande $=0.50$ para pruebas no paramétricas . Además, se calculó la potencia estadística (1- $\beta$ ), es decir, la capacidad de la prueba para detectar diferencias (mínimo 0.80) ). $^{26}$

Previamente se pidió el consentimiento informado a cada participante, explicando los objetivos del estudio, se garantizó que no corrían ningún riesgo durante la intervención, puntualizando además la libertad de dejar de participar cuando lo consideraran sin ningún compromiso. Se contó además con la aprobación del comité de ética de la Facultad de Enfermería donde está la sede del Proyecto (Dictamen: No. 004-2019) y de las instituciones participantes (Ministerio de Salud e Instituto Nacional de Enfermedades Neoplásicas, Perú).

\section{RESULTADOS}

Con respecto al nivel educativo, y pese a que existen diferencias entre los tres grupos, todos ellos presentan una media por encima de 10 años. El grupo de diabetes duplica en porcentaje al presentar más de una enfermedad crónica con 41\%, a diferencia de los grupos con diabetes e hipertensión. En los tres grupos de estudio las mujeres son mayoría. El promedio de edad difiere entre grupos, las personas de menor edad pertenecen al grupo con cáncer con una media de 56 años, en contraste con los otros dos grupos que registraron de 60 a más años. Para el tipo de seguro de atención médica, 94\% del grupo con diagnóstico de cáncer refiere tener seguro popular o del gobierno, a diferencia de los otros dos grupos donde un tercio de ellos cuentan con seguro privado. Los tres grupos presentan más de 5 años viviendo con la enfermedad. Todos manifiestan pertenecer a familias compuestas de 4 a 5 miembros (Tabla 3).

Tabla 3. Datos sociodemográficos y condiciones de salud

\begin{tabular}{|c|c|c|c|c|c|c|c|}
\hline \multirow[t]{2}{*}{ Variable } & \multicolumn{2}{|c|}{ Diabetes } & \multicolumn{2}{|c|}{ Hipertensión } & \multicolumn{2}{|c|}{ Cáncer } & \multirow[t]{2}{*}{ Sig. } \\
\hline & (187 & $100 \%)$ & $(215$ & $100 \%)$ & $(160$ & $100 \%)$ & \\
\hline & \# & $\%$ & \# & $\%$ & \# & $\%$ & \\
\hline Femenino & 113 & $60.4 \%$ & 138 & $64.2 \%$ & 104 & $65.0 \%$ & .628 \\
\hline Masculino & 74 & $39.6 \%$ & 77 & $35.8 \%$ & 56 & $35.0 \%$ & \\
\hline Edad & \multicolumn{2}{|c|}{$\mu: 60.82$} & \multicolumn{2}{|c|}{$\mu: 63.27$} & \multicolumn{2}{|c|}{$\mu: 55.97$} & .001 \\
\hline Años de educación & \multicolumn{2}{|c|}{$\mu: 11.10$} & \multicolumn{2}{|c|}{$\mu: 10.67$} & \multicolumn{2}{|c|}{$\mu: 9.71$} & .006 \\
\hline $\begin{array}{l}\text { Número de miembros de } \\
\text { familia }\end{array}$ & \multicolumn{2}{|c|}{$\mu: 4.51$} & \multicolumn{2}{|c|}{$\mu: 4.53$} & \multicolumn{2}{|c|}{$\mu: 4.95$} & .101 \\
\hline \multicolumn{8}{|c|}{ Número de enfermedades crónicas: } \\
\hline Una & 110 & $58.8 \%$ & 167 & $77.7 \%$ & 125 & $78.1 \%$ & .001 \\
\hline Más de una & 77 & $41.2 \%$ & 48 & $22.3 \%$ & 35 & $21.9 \%$ & \\
\hline \multicolumn{8}{|l|}{ Tipo de seguro de salud: } \\
\hline Seguro Popular & 125 & $66.8 \%$ & 148 & $68.8 \%$ & 150 & $93.8 \%$ & .001 \\
\hline Seguro Privado & 62 & $33.2 \%$ & 67 & $31.2 \%$ & 10 & $6 \%$ & \\
\hline Años con la enfermedad & \multicolumn{2}{|c|}{$\mu: 7.63$} & \multicolumn{2}{|c|}{$\mu: 8.14$} & \multicolumn{2}{|c|}{$\mu: 5.95$} & .035 \\
\hline
\end{tabular}

Nota: $p<0.05$ 
En relación con los resultados del automanejo, la dimensión del automanejo global (con valores mínimo 0 y máximo 96) registró puntaje mínimo de 3 y máximo de 96 con una media de 66 puntos (Tabla 4). Los resultados según el diagnóstico médico muestran diferencias estadísticamente significativas ( $p=0.001$ ) entre el grupo de diabetes vs cáncer e hipertensión vs cáncer, pues el grupo de cáncer presenta 10 puntos más que los otros dos grupos. Sin embargo, el efecto de estas diferencias debido al diagnóstico de la enfermedad tiene un valor $\mathrm{d}=0.30$, considerando una potencia estadística 1- $\beta .99$ (Tabla 5).

Tabla 4. Diferencias del automanejo según diagnóstico médico

\begin{tabular}{|c|c|c|c|c|}
\hline \multirow{4}{*}{$\begin{array}{l}\text { Variables } \\
\text { Índice general } \\
(0-96)\end{array}$} & \multirow{4}{*}{$\begin{array}{c}\text { Población General } \\
\text { (n: 562) } \\
\text { Media } \\
66.18 \\
(3-96)\end{array}$} & \multirow{2}{*}{$\begin{array}{l}\text { Diagnóstico } \\
\text { Diabetes }\end{array}$} & \multicolumn{2}{|c|}{ Media } \\
\hline & & & 63.16 & $(3-96)$ \\
\hline & & Hipertensión & 63.63 & (11-96) \\
\hline & & Cáncer & 73.13 & $(24-96)$ \\
\hline \multirow{3}{*}{$\begin{array}{l}\text { Conocimiento } \\
(0-16)\end{array}$} & \multirow{3}{*}{$\begin{array}{l}10.38 \\
(0-16)\end{array}$} & Diabetes & 10.24 & $(0-16)$ \\
\hline & & Hipertensión & 10.05 & $(1-16)$ \\
\hline & & Cáncer & 10.99 & $(0-16)$ \\
\hline \multirow{3}{*}{$\begin{array}{l}\text { Manejo del impacto en aspectos físicos, } \\
\text { emocionales y sociales } \\
(0-24)\end{array}$} & \multirow{3}{*}{$\begin{array}{l}16.67 \\
(1-24)\end{array}$} & Diabetes & 15.81 & $(1-24)$ \\
\hline & & Hipertensión & 16.02 & $(3-24)$ \\
\hline & & Cáncer & 18.54 & $(6-24)$ \\
\hline \multirow{3}{*}{$\begin{array}{l}\text { Automanejo de las condiciones médicas } \\
(0-56)\end{array}$} & \multirow{3}{*}{$\begin{array}{l}39.13 \\
(0-56)\end{array}$} & Diabetes & 37.11 & $(0-56)$ \\
\hline & & Hipertensión & 37.57 & $(4-56)$ \\
\hline & & Cáncer & 43.59 & $(14-56)$ \\
\hline
\end{tabular}

Cáncer n:160; Hipertensión n:215; Diabetes n:187

Los resultados según la dimensión de conocimiento presentan un rango de 0-16 con una media de 10.38 puntos (Tabla 4), las diferencias de acuerdo con el diagnóstico médico muestran resultados similares al automanejo global, esto es, diferencias similares en el grupo de diabetes e hipertensión y diferencias estadísticamente significativas entre el grupo diabetes vs cáncer e hipertensión vs cáncer ( $p<0.005)$. Sin embargo, el efecto de estas diferencias debido al diagnóstico médico es bajo d=0.11 y presentan una potencia estadística 1- $\beta .46$ (Tabla 5).

Tabla 5. Diferencias del automanejo según diagnóstico médico

\begin{tabular}{llrrrrr} 
& \multicolumn{1}{c}{ Variable } & $\begin{array}{c}\text { Test } \\
\text { Statistic }\end{array}$ & $\begin{array}{c}\text { Std. } \\
\text { Error }\end{array}$ & sig & 1- $\boldsymbol{\beta}$ & D \\
\hline Índice & Diabetes-Hipertensión & -8.68 & 16.23 & .592 & .99 & .32 \\
\cline { 2 - 7 } General & Diabetes-Cáncer & -107.47 & 17.48 & .001 & & \\
\cline { 2 - 7 } & Hipertensión-Cáncer & -98.79 & 16.94 & .001 & & .11 \\
\hline Conocimiento & Diabetes-Hipertensión & 3.69 & 16.15 & .819 & .46 & \\
\cline { 2 - 7 } & Diabetes-Cáncer & -48.05 & 16.86 & .013 & & \\
\cline { 2 - 7 } & Hipertensión-Cáncer & -44.35 & 17.39 & .032 & & \\
\hline
\end{tabular}




\begin{tabular}{|c|c|c|c|c|c|c|}
\hline \multirow{3}{*}{$\begin{array}{l}\text { Manejo del impacto } \\
\text { en aspectos físicos, } \\
\text { emocionales y sociales }\end{array}$} & Diabetes-Hipertensión & -9.269 & 16.18 & .567 & .04 & .03 \\
\hline & Diabetes-Cáncer & -109.341 & 17.43 & .001 & & \\
\hline & Hipertensión-Cáncer & -99.07 & 16.90 & .001 & & \\
\hline \multirow{3}{*}{$\begin{array}{l}\text { Automanejo de las } \\
\text { condiciones médicas }\end{array}$} & Diabetes-Hipertensión & -10.95 & 16.22 & .500 & .99 & .28 \\
\hline & Diabetes-Cáncer & -109.88 & 17.47 & .001 & & \\
\hline & Hipertensión-Cáncer & -99.92 & 16.94 & .001 & & \\
\hline
\end{tabular}

Los resultados de la dimensión manejo del impacto en aspectos físicos, emocionales y sociales presenta rangos de 1-24 puntos, registra una media de 16.67, con resultados similares a la dimensión de conocimiento y valores de efecto de las diferencias bajas $d=0.03$ y una potencia estadística $1-\beta$ .04 (tabla 5). La dimensión automanejo de la adherencia al cuidado médico presenta una media de 39.13 con rangos de 0-56. De igual forma, presenta diferencias entre grupos de diabetes vs cáncer, hipertensión vs cáncer; en los grupos de diabetes e hipertensión no se presentan diferencias estadísticamente significativas con valores de efecto de las diferencias bajas $\mathrm{d}=0.28$ y una potencia estadística 1- $\beta .99$ (Tabla 5).

\section{DISCUSIÓN}

De conformidad con uno de los propósitos del presente estudio de describir el automanejo en personas con enfermedades crónicas diferentes, los resultados muestran que el automanejo aún es deficiente en personas con alguna condición crónica en la población peruana, datos similares a estudios de años anteriores ${ }^{27,28}$, los cuales presentan una media de automanejo global de 66 puntos, con un puntaje máximo de 96 . A su vez, se encuentran resultados semejantes en estudios realizados en población mexicana ${ }^{29-31} \mathrm{y}$ colombiana ${ }^{32}$, con resultados en las diferentes dimensiones del automanejo: conocimientos de su salud y enfermedad, manejo del impacto de su enfermedad en aspectos físicos, emocionales y sociales, en la adherencia a los cuidados médicos (adherencia al tratamiento, cumplimento de las indicaciones médicas como el tratamiento médico y seguimiento en dieta, ejercicio, además de la comunicación con los proveedores de salud, en especial la consulta médica).

Asimismo, se reconoce la importancia del automanejo en personas con cronicidad ${ }^{33}$, evidenciando diferencias en personas que cuidan de manera adecuada su enfermedad con respecto a las que no lo hacen, según resultados de evaluaciones de programas que promueven el automanejo con resultados favorables en la salud de los participantes ${ }^{34-36}$, sobre todo en los aspectos emocionales ${ }^{37.38}$. Cabe destacar que en Latinoamérica sólo se encontró un estudio con resultados favorables en México²0.

Por otra parte, la Organización Panamericana de la Salud considera el automanejo como una estrategia prometedora de atención de las enfermedades crónicas que permite ir más allá de la información, para enseñar activamente a las personas a detectar y resolver los problemas asociados con la enfermedad que tienen ${ }^{33}$. Por consiguiente, el reto es grande para Latinoamérica, empezando con el abordaje en la formación del recurso humano en salud con un cambio en el enfoque de la atención y educación en salud, dando al paciente el rol protagónico en la atención, y así realizar un trabajo colaborativo con la persona que vive con una enfermedad crónica ${ }^{39}$.

Con relación al análisis de las diferencias o similitudes del automanejo entre grupos con diferente diagnóstico (diabetes, hipertensión y cáncer), los resultados muestran pocas diferencias a tener en cuenta, solo en el índice global se podría considerar diferencias derivadas del diagnóstico médico con un valor medio $(\mathrm{d}=0.30)$. Un resultado distinto se obtuvo en un estudio realizado también en 
Perú ${ }^{27}$, donde encontraron diferencias significativas en la dimensión de manejo del impacto de la enfermedad entre grupo de diabetes vs el grupo de cáncer, siendo mejor en el grupo de diabetes; asimismo, se observaron diferencias significativas en la dimensión de cuidados médicos entre ambos grupos, en el grupo de cáncer estuvo mejor valorado. Sin embargo, cabe señalar que no se analizó si el efecto de estas diferencias se derivaba por el diagnóstico y la potencia estadística del estudio.

Estos resultados nos llevan a valorar los programas genéricos que promueven el automanejo, como son el programa de Stanford ${ }^{40}$ y el Programa Flinders ${ }^{41}$, sin dejar de valorar la existencia de programas específicos por enfermedad42-44. No obstante, pensando en el primer nivel de atención, consideramos que estos programas tendrían un rol muy importante donde la atención es a todo tipo de población.

Cabe señalar que los resultados presentados deben ser considerados solo representativos para la población de estudio, dado que no se pudo realizar una muestra probabilística. Sin embargo, consideramos que estos visibilizan la necesidad de seguir realizando más investigaciones en población peruana y en Latinoamérica, pues aún es muy escaso el conocimiento relacionado con el automanejo en nuestros países. Por ende, las investigaciones en dicha área deberían de incrementar, y más aún en personas con cáncer, sugerencia emitida por la Sociedad de Enfermería Oncológica de los Estados Unidos ${ }^{45}$.

Asimismo, se reafirma la necesidad de fortalecer el rol educativo por parte de los profesionales de la salud, con este nuevo enfoque de educación, en especial el de enfermería, quien tiene un rol protagónico en esta área relacionada con la formación en salud y sobre todo en el primer nivel de atención, en concordancia con lo sugerido por la Organización Mundial de la Salud como un eje prioritario para la región ${ }^{33}$.

\section{CONCLUSIONES}

Los resultados muestran que las personas con alguna condición crónica presentan un automanejo inadecuado, no presentan diferencias en el automanejo global y sus dimensiones según diagnóstico médico, por lo que se sugiere la implementación de programas genéricos que promuevan el automanejo, sobre todo en el primer nivel de atención. De igual forma, se debe incrementar el número de investigaciones en Latinoamérica, con mayor atención en personas con cáncer.

\section{RESPONSABILIDADES ÉTICAS}

Protección de personas y animales. Los autores declaran que los procedimientos realizados se ajustaron a la Declaración de Helsinki y a la Ley General de Salud de México en materia de investigación. Confidencialidad. Los autores declaran que han obtenido el consentimiento informado de los sujetos referidos en el estudio; asimismo, los datos confidenciales fueron protegidos conforme al reglamento de la Ley General de Salud en Materia de Investigación para la Salud.

Conflicto de intereses. Los autores declaran no tener conflicto de intereses.

Financiamiento. Este proyecto fue financiado por CONACYT: Red temática de Automanejo en crónicos \#270842

Agradecimientos. Agradecemos el apoyo técnico y económico en la realización del presente estudio a la Universidad Autónoma de Tamaulipas, Facultad de Enfermería Campus Tampico; asimismo, al Instituto Nacional de Enfermedades Neoplásicas-Perú y a la Dirección de la Red de Servicios de Salud Lima-Norte-Ministerio de Salud de Perú. 


\section{REFERENCIAS}

1. Legetic B, Medici A, Hernández-Ávila M, Alleyne GAO, Hennis A (Ed.) / Organización Panamericana de la Salud. Las dimensiones económicas de las enfermedades no transmisibles en América Latina y el Caribe. $3^{\text {th }}$ ed. Washington, D.C.: OPS; 2017. https://bit.ly/3h7eVXU

2. Ministerio de Salud Perú. Plan Esperanza Memoria. Perú: Minsa; 2015. http://bitly.ws/e4PT

3. Seclen SN, Rosas ME, Arias AJ, Huayta E, Medina CA. Prevalence of diabetes and impaired fasting glucose in Peru: Report from PERUDIAB, a national urban population-based longitudinal study. BMJ Open Diabetes Res Care. 2015; 3(1): 1-7. https://doi.org/10.1136/bmjdrc-2015-000110

4. Revilla L, López T, Sánchez S, Yasuda M, Sanjinés G. Prevalencia de hipertensión arterial y diabetes en habitantes de Lima y Callao, Perú. Rev. Peru. Med. Exp. Salúd pública. 2014; 31(3): 437-44.

https://doi.org/10.17843/rpmesp.2014.313.78

5. Instituto Nacional de Estadística e Informática (INEI). Perú Enfermedades no transmisibles y transmisibles 2017. Perú: INEI; 2017. http://bitly.ws/e4TI

6. Fernández-Cantón SB. La atención centrada en el paciente: una visión general sobre su enfoque en los servicios de salud. Boletín CONAMED. 2019; 4(23): 13-23. http://bitly.ws/e4Vp

7. Frost J, Garside R, Cooper C, Britten N. A qualitative synthesis of diabetes self-management strategies for long term medical outcomes and quality of life in the UK. BMC Health Serv Res. 2014; 14: 1-15. https://doi.org/10.1186/1472-6963-14-348

8. Corbin J, Strauss A. Managing chronic illness at home: Three lines of work. Qual Sociol. 1985; 8: 224-47. https://doi.org/10.1007/BFoog89485

9. Institute of Medicine (US) Committee on the Crossing the Quality Chasm: Next Steps Toward a New Health Care System. The 1st annual crossing the quality chasm summit: A focus on communities. Washington, D.C.: National Academies Press (US); 2004. https://doi.org/10.17226/11085

10. Satink T, Josephsson S, Zajec J, Cup EHC, de Swart BJM, Nijhuis-van der Sanden MWG. Negotiating role management through everyday activities: Narratives in action of two stroke survivors and their spouses. Disabil Rehabil. 2016; 38(24): 2354-64.

https://doi.org/10.3109/09638288.2015.1129442

11. Satink T, Josephsson S, Zajec J, Cup EHC, de Swart BJM, Nijhuis-van der Sanden MWG. Self-management develops through doing of everyday activities-a longitudinal qualitative study of stroke survivors during two years post-stroke. BMC Neurol. 2016; 16:1-13.

https://doi.org/10.1186/s12883-016-0739-4

12. Vernooij RWM, Willson M, Gagliardi AR, Members of the Guidelines International Network Implementation Working Group. Characterizing patient-oriented tools that could be packaged with guidelines to promote self-management and guideline adoption: A meta-review. Implement Sci. 2016; 11(52): 1-13. https://doi.org/10.1186/s13012-016-0419-1

13. Pearce G, Parke HL, Pinnock H, Epiphaniou E, Bourne CLA, Sheikh A, et al. The PRISMS taxonomy of self-management support: Derivation of a novel taxonomy and initial testing of its utility. J Health Serv Res Policy. 2016; 21(2): 73-82. https://doi.org/10.1177/1355819615602725

14. Audulv A, Ghahari S, Kephart G, Warner G, Packer TL. The Taxonomy of Everyday Self-management Strategies (TEDSS): A framework derived from the literature and refined using empirical data. Patient Educ Couns. 2019; 102(2): 367-75. https://doi.org/10.1016/j.pec.2018.08.034

15. Schulman-Green D, Jaser S, Faith M, Alonzo A, Grey M, McCorkle R, et al. Processes of self-management in chronic illness. J. Nurs Scholarsh. 2012; 44(2): 136-44.

https://doi.org/10.1111/j.1547-5069.2012.01444.x 
16. Ryan P, Sawin KJ. The individual and family self-management theory: Background and perspectives on context, process, and outcomes. Nurs Outlook. 2009; 57(4): 217-25.

https://doi.org/10.1016/j.outlook.2008.10.004

17. Battersby MW. Health reform through coordinated care: SA HealthPlus. BMJ. 2005; 330(7492): 662-5. https://doi.org/10.1136/bmj.330.7492.662

18. Battersby M, Ask A, Reece MM, Markwick MJ, Collins J. The partners in health scale: The development and psychometric properties of a generic assessment scale for chronic condition self-management. Aust J Prim Health. 2003; 9(4): 41-52. https://doi.org/10.1071/PY03022

19. Lorig KR, Holman H. Self-management education: History, definition, outcomes, and mechanisms. Ann Behav Med. 2003; 26(1): 1-7. https://doi.org/10.1207/S15324796ABM2601_01

Peñarrieta-de Córdova MI, Leon R, Gutierrez T, Mier N, Banda O, Delabra M. Effectiveness of a chronic disease self-management program in Mexico: A randomized controlled study. J Nurs Educ Pract. 2017; 7(7): 87-94. https://doi.org/10.5430/jnep.v7n7p87

21. Lorig KR, Sobel DS, Ritter PL, Laurent D, Hobbs M. Effect of a self-management program on patients with chronic disease. Eff Clin Pract. 2001; 4(6): 256-62. https://bit.ly/3hadOXr

22. Peñarrieta-de Córdova M, Vergel-Camacho O, Schmith-Álvarez L, Lezama-Vigo S, Rivero-Álvarez $\mathrm{R}$, Taipe-Cancho J, et al. Validación de un instrumento para evaluar el automanejo en enfermedades crónicas en el primer nivel de atención en salud. Rev. Cient. de Enferm. 2012; VIII(1): 64-73. https://bit.ly/3j6raUF

23. Hudon É, Chouinard MC, Krieg C, Lambert M, Joober H, Lawn S, et al. The french adaptation and validation of the Partners in Health (PIH) scale among patients with chronic conditions seen in primary care. PLoS One. 2019; 14(10): 1-11. https://doi.org/10.1371/journal.pone.0224191

24. Chiu TML, Tam KTW, Siu CF, Chau PWP, Battersby M. Validation study of a Chinese version of Partners in health in Hong Kong (C-PIH HK). Oual Life Res. 2017; 26(1):199-203.

https://doi.org/10.1007/s11136-016-1315-5

25. Veldman K, Reijneveld SA, Lahr MMH, Uittenbroek RJ, Wynia K. The Partners in Health scale for older adults: Design and examination of its psychometric properties in a Dutch population of older adults. Health Expect. 2016; 20(4): 601-7. https://doi.org/10.1111/hex.12488

26. Cárdenas-Castro M, Arancibia-Martini H. Potencia estadística y cálculo del tamaño del efecto en G*Power: complementos a las pruebas de significación estadística y su aplicación en psicología. Salud soc. 2014; 5(2): 210-24. https://doi.org/10.22199/So7187475.2014.0002.00006

27. Peñarrieta-de Córdova MI, Reyes G, Krederdt S, Flores F, Resendiz E, Chávez-Flores E. Automanejo en enfermedades crónicas: diabetes mellitus tipo 2, hipertensión arterial y cáncer. Revista de Investigación UNW. 2015; (4): 43-56. https://bit.ly/352QZZOP

28. Lagos-Méndez H, Flores-Rodríguez N. Funcionalidad familiar y automanejo de pacientes con diabetes mellitus e hipertensión arterial en el Hospital de Puente Piedra-Lima. Cuid salud. 2014; 1(2): 85-92. https://doi.org/10.31381/cuidado_y_salud.v1i2.1111

29. Mar-García J, Peñarrieta-de Córdova I, León-Hernández R, Gutiérrez-Gómez T, Banda-González $\mathrm{O}$, Rangel-Torres S, et al. Relación entre automanejo y percepción de funcionalidad familiar en personas con diabetes mellitus tipo 2. Enferm. univ. 2017; 14(3): 155-61.

http://dx.doi.org/10.1016/j.reu.2017.05.005

30. León-Ramírez MS, Peñarrieta-de Córdova MI, León-Hernández R, Gutiérrez-Gómez T, Piñones-Martínez MS, Quintero-Valle LM. Relación entre automanejo y síntomas depresivos en personas con diabetes e hipertensión. Enferm. univ. 2020; 17(1): 5-15.

https://doi.org/10.22201/eneo.23958421e.2020.1.681 
31. Díaz-Rentería MG, Díaz-Díaz GJ, Soto-García AN, Campa-Aguilera KC, Ulloa-Ruvalcaba N, Medina-González MG, et al. Nivel de automanejo en adultos mayores con enfermedades crónicas no transmisibles. RevSalJal. 2020; (Núm. Esp.): 31-7. https://bit.ly/3pLqVRO

32. Vergara-Escobar OJ. Intervención de enfermería para el automanejo en adultos con cáncer colorrectal en tratamiento quirúrgico. [Tesis Doctorado]. Bogotá: Universidad Nacional de Colombia; 2020. https://bit.ly/3AjokAa

33. Organización Panamericana de la Salud/Organización Mundial de la Salud. Cuidados innovadores para las condiciones crónicas: organización y prestación de atención de alta calidad a las enfermedades crónicas no transmisibles en las Américas. Washington, D.C.: OPS/OMS; 2013. https://bit.ly/3zeXiwu

34. Lorig K, Ritter PL, Turner RM, English K, Laurent DD, Greenberg J. A diabetes self-management program: 12-month outcome sustainability from a nonreinforced pragmatic trial. J Med Internet Res. 2016; 18(12): 1-10. https://doi.org/10.2196/jmir.6484

35. Harvey PW, Petkov JN, Misan G, Fuller J, Battersby MW, Cayetano TN, et al. Self-management support and training for patients with chronic and complex conditions improves health-related behaviour and health outcomes. Aust Health Rev. 2008; 32(2): 330-8.

https://doi.org/10.1071/aho80330

36. Kim SH, Kim K, Mayer DK. Self-management intervention for adult cancer survivors after treatment: A systematic review and meta-analysis. Oncol Nurs Forum. 2017; 44(6): 719-28.

https://doi.org/10.1188/17.ONF.719-728

37. Pal K, Eastwood SV, Michie S, Farmer AJ, Barnard ML, Peacock R, et al. Computer-based diabetes self-management interventions for adults with type 2 diabetes mellitus. Cochrane Database Syst Rev. 2013; 2013(3): 1-114. https://bit.ly/2UZGJVG

38. Cheng KKF, Lim YTE, Koh ZM, Tam WWS. Home-based multidimensional survivorship programmes for breast cancer survivors. Cochrane Database Syst Rev. 2017; 8(8): 1-118. https://cutt.ly/bmflXLf

39. McDonald KM, Sundaram V, Bravata DM, Lewis R, Lin N, Kraft SA, et al. Closing the quality gap: A critical analysis of quality improvement strategies (Vol. 7: Care coordination). Rockville (MD), EE.UU.: Agency for Healthcare Research and Ouality (US); 2007. https://bit.ly/2UTOyvX

40. Lorig KR, Ritter PL, González VM. Hispanic chronic disease self-management: A randomized community-based outcome trial. J Nurs Res. 2003; 52(6): 361-9.

https://doi.org/10.1097/00006199-200311000-00003

41. Battersby M, Harris M, Smith D, Reed R, Woodman R. A pragmatic randomized controlled trial of the Flinders Program of chronic condition management in community health care services. Patient Educ Couns. 2015; 98(11): 1367-75. https://doi.org/10.1016/j.pec.2015.06.003

42. Boland L, Bennett K, Connolly D. Self-management interventions for cancer survivors: A systematic review. Support Care Cancer. 2018; 26(5): 1585-95. https://doi.org/10.1007/so0520-017-3999-7

43. Mileski M, Kruse CS, Catalani J, Haderer T. Adopting telemedicine for the self-management of hypertension: Systematic review. JMIR Med Inform. 2017; 5(4): 1-12.

https://doi.org/10.2196/medinform.6603

44. Hoffman AJ. Enhancing self-efficacy for optimized patient outcomes through the theory of symptom self-management. Cancer Nurs. 2013; 36(1): 16-26.

https://doi.org/10.1097/NCC.obo13e31824a730a

45. Doorenbos AZ, Berger AM, Brohard-Holbert C, Eaton L, Kozachik S, LoBiondo-Wood G, et al. 2008 ONS research priorities survey. Oncol Nurs Forum. 2008; 35(6): 100-7.

https://doi.org/10.1188/o8.onf.e10o-e107 